\title{
Data Analytics Framework for A Game-based Rehabilitation System
}

\author{
Jiongqian Liang ${ }^{\dagger *}$, David Fuhry ${ }^{\dagger}$, David Maung ${ }^{\dagger}$, Alexandra Borstad ${ }^{\ddagger}$, Roger Crawfis ${ }^{\dagger}$, \\ Lynne Gauthier ${ }^{\ddagger}$, Arnab Nandi ${ }^{\dagger}$ and Srinivasan Parthasarathy ${ }^{\dagger *}$ \\ ${ }^{\dagger}$ Computer Science and Engineering, The Ohio State University, Columbus, $\mathrm{OH}$ \\ ${ }^{\ddagger}$ College of Medicine, The Ohio State University, Columbus, $\mathrm{OH}$ \\ *Contact: \{liangji, srini\}@cse.ohio-state.edu
}

\begin{abstract}
Stroke is a major cause of hemiparesis in United States. Constraint-Induced Movement therapy (CI therapy) is an effective treatment for upper extremity hemiparesis; however it is inaccessible to most patients. To make it more accessible, we developed a game-based rehabilitation system incorporating the major rehabilitation principles from CI therapy. We introduce a data analytics framework for our rehabilitation system in this paper that can provide objective measures of motor performance during gameplay. We design techniques of preprocessing collected data and propose a series of kinematic measurements, which are used to assess the motor performance and supplement in-clinic measures of therapeutic effect. We also present contextual filtering techniques to enable comparing movement production under different conditions, e.g., self-paced versus gamepaced movement. We apply our data analytics framework on data collected from several participants. Our analysis shows that participants' motor movement improves over the period of treatment, with different participants showing different patterns of improvement, e.g., speed versus range of motion. Results of kinematic measurements during gameplay are highly consistent with in-clinic performance based on the Wolf Motor Function Test. Moreover, our fine-grained trend analysis reveals potential to detect fatigue, which is related to the duration of gameplay.
\end{abstract}

\section{Keywords}

Game-based rehabilitation, Data analytics framework, Kinematic measurement, Therapy efficacy, Fatigue.

\section{INTRODUCTION}

As one main cause of serious and long-term disability [3], it is estimated that there are more than 4 million people with upper extremity disability in the US, and $26 \%$ of them need others' assistance in daily life $[9,6,2]$. The expense of caring for these stroke survivors and accompanying loss

Permission to make digital or hard copies of all or part of this work for personal or classroom use is granted without fee provided that copies are not made or distributed for profit or commercial advantage and that copies bear this notice and the full citation on the first page. Copyrights for components of this work owned by others than the author(s) must be honored. Abstracting with credit is permitted. To copy otherwise, or republish, to post on servers or to redistribute to lists, requires prior specific permission and/or a fee. Request permissions from permissions@acm.org.

DH '16, April 11 - 13, 2016, Montréal, QC, Canada

(C) 2016 Copyright held by the owner/author(s). Publication rights licensed to ACM. ISBN 978-1-4503-4224-7/16/04 . .\$15.00

DOI: http://dx.doi.org/10.1145/2896338.2896356 of productivity costs the US economy more than $\$ 34$ billion annually [12]. Moreover, only $30.7 \%$ receive outpatient rehabilitation services, the majority of whom do not receive treatments with strong empirical evidence of efficacy [4]. More effective rehabilitation approaches, such as ConstraintInduced Movement therapy (CI therapy) [14], are costly and difficult for patients to access.

In an attempt to address this issue, we collaborated with the stroke community to design a home-based rehabilitation game system [11]. The system uses advanced and commercially available gaming technology, and consists of a computer, a Microsoft KinectOne camera, the rehabilitation software, and biofeedback of arm use through a smart watch application. The interactive computer game adopts the critical principles of CI therapy. Participants were asked to play the game for 30 hours over the course of 3 weeks, during which the game and movement data was collected.

Although Kinect game-based rehabilitation systems have been developed by others [8, 7], to our knowledge none provides knowledge of results based on kinematic data collected during gameplay. Su et al. develop exercise assistant software wherein a participant repeats movements previously performed under a clinical professional's supervision, and performance is assessed for four people [13]. However, their system encourages in-home users to reproduce the same movement patterns that they performed under therapist supervision and does not comprise a game. Chang et al [1] showed that their interactive rehabilitation system for young adults outperformed participants following verbal instructions of a therapist. But their study was limited to two participants and reported only the number of correct movements each day.

We expand on this prior work by evaluating the kinematic improvements in eight participants with Multiple Sclerosis (MS), each of whom performs in a game-based environment that adapts its difficulty to their performance. In addition, our system includes a data analytics component to demonstrate the feasibility of evaluating the effect of rehabilitation using a subset of kinematic variables, to assess participant fatigue, and to demonstrate that participants can respond differently in terms of movement quality to alterations in the pace of gameplay (self paced versus rapid pace promoted through the game).

In this paper, we introduce a data analytics framework to analyze the efficacy of our rehabilitation system on participants. Specifically, we design data preprocessing techniques to smooth the skeleton data obtained from Kinect sensors and remove anomalies. We provide contextual fil- 


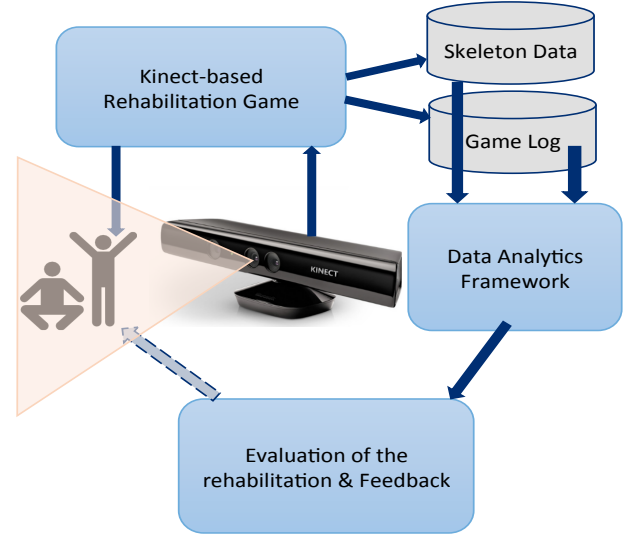

Figure 1: Rehabilitation System Overview

tering to study performance under different conditions (e.g., self-paced versus fixed pace). Segmentation of sessions enables us to study the change of performance through movement trend analysis within and across sessions (e.g., fatigue detection). To evaluate the motor performance of users in the gameplay, we propose three kinematic measurements from different aspects: hand speed, angle speed and range of motion. By running our data analytics framework on the data we collected from participants, we observe the results of our kinematic measurements are greatly consistent with popular Wolf Motor Function Test [5] on measuring the strength of motor performance, improvement trend and scale. Our two-half comparison shows that majority of the participants benefit from the rehabilitation game system, but might be in different ways, either hand speed, angle speed or range of motion. We finally conduct a fine-grained movement trend analysis, where we find the presence of fatigue in gameplay. We further show that the fatigue phenomenon is particularly related with the amount of gameplay time.

The data analytics framework can improve clinical care in several ways: 1) Provide an objective measure of improvement without requiring time-costly clinical tests. 2) Enable assessment of dose-response to rehabilitation (e.g., kinematic data is continuously collected versus intermittently assessed clinical outcome measure). 3) Assessment can be done remotely without a therapist present.

The paper is organized as follows. Section 2 provides an overview of the rehabilitation system. Section 3 presents our data analytics framework in detail. Section 4 shows our analysis results on data collected from participant in-home gameplays. We conclude in Section 5 .

\section{SYSTEM OVERVIEW}

Here we briefly introduce the structure of the rehabilitation system, termed Recovery Rapids, and discuss how the data analytics framework interacts with other components. Figure 1 shows the overview of the rehabilitation system.

Collaborating with the stroke community, we developed the rehabilitation game system composed of a computer, Microsoft Kinect Camera and the rehabilitation software. In Figure 1, the left part shows the participant and the game software. The participant plays the game interactively in front of the computer and Kinect Sensors con- tinuously record the position of 20 skeletal joints. In the game, the user controls an Avatar and navigates a kayak down a river canyon that winds through various terrains (mountains, tropical, desert, underground caves). The user is required to collect hidden treasure, fish for food, capture essential supplies, gather food along the river banks, and pull objects from the river (for more information about the game scene, refer to the video ${ }^{1}$ ). Before playing the game, the therapist can specify the relative frequency of various game mechanics (which prompt various movements) to obtain a therapy program that is customized to a person's motor presentation. Procedural content generation [11] is employed to generate the game environment for each user based on user's motor presentation.

The game collects fine-grained skeleton data of the user using the Kinect sensors at a frequency of $30 \mathrm{~Hz}$. The Kinect sensors track 20 joints of the body, including the shoulder, elbow, wrist, hand, and hip center, which are most relevant to this application. Each entry in the skeleton data, called a snapshot, contains the $3-\mathrm{D}$ coordinates of these 20 positions with a timestamp. The game also matches the skeleton data to coded gestures in real time, each of which contains a series of states [10]. If the movement of the user matches a state (such as "begin" or "end") of a coded gesture, then the gesture and state is then stored into the game log with a timestamp. If the movement being performed meets all the continuous states of a gesture, a game mechanic is triggered and a timestamped record of the therapeutic movement (e.g., fishing) is kept in the game log. Therefore, as shown in upper right corner in Figure 1, the system outputs two categories of data, skeleton data and game log. Note that both the skeleton data and game log contain timestamps, so it is possible to jointly analyze them.

For the purpose of treatment, the game automatically adjusts the difficulty of each movement based on the participant's performance. For example, if a larger movement is consistently recognized, the game will no longer accept smaller attempts. Therefore, the user is motivated to continually perform to his/her maximal ability. Moreover, the user encounters varying environments with varying levels of challenge (e.g., being prompted to alternate between different movements versus continually produce the same movement) and varying pace. Some sections of the game promote fast movements at a fixed pace established in the game (e.g., Rapids Region) while others allow self-paced movements (e.g., Rowing Region).

Here, we propose a data analytics framework integrated within the game software to understand and evaluate the motor performance of participants. As shown in Figure 1, the data analytics framework takes the skeleton data and game log as input, and outputs the evaluation of the rehabilitation. The evaluation result can then be presented to users and clinical staff to provide an objective measure of motor performance over time. We describe the data analytics framework in detail in the next section.

\section{DATA ANALYTICS FRAMEWORK}

In this section, we describe the data analytics framework of our rehabilitation system. As shown in Figure 2, our data analytics framework consists of data preprocessing, contextual filtering and rehabilitation analysis.

\footnotetext{
$\overline{{ }^{1} \text { http://youtu.be/uAysIGueN }} 9 \mathrm{U}$
} 


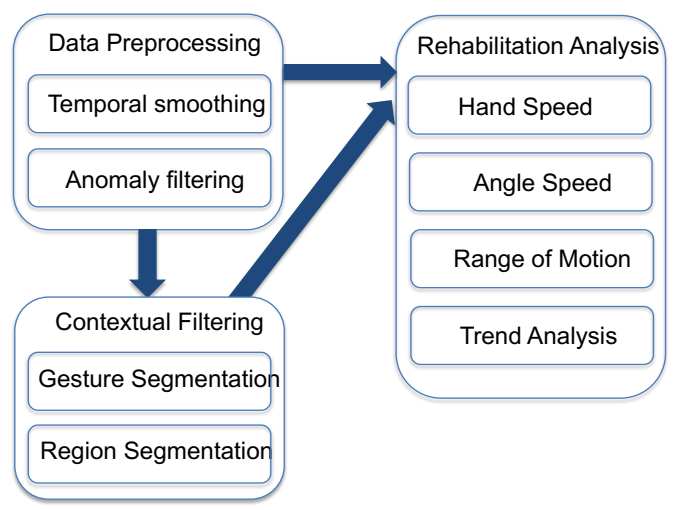

Figure 2: Data Analytics Framework

\subsection{Data Preprocessing}

Before we analyze the collected data, we need to preprocess it to correct several problems which occur particularly with the skeleton data. The Kinect sensors collect data at a frequency as high as $30 \mathrm{~Hz}$. Though this provides fair temporal granularity, two main problems remain with the raw data which we correct with the below operations.

Temporal smoothing: One issue is that a pair of contiguous snapshots may be so close in time that most of the change in their skeleton coordinates might not be due to the user's movement, but instead by random errors (i.e., "jitter") resulting from limited sensor spatial resolution. Though the errors can be as small as a few millimeters, the sensors collect 30 snapshots every second and accumulating these errors over time can adversely affect the analytics result. To tackle this problem, we smooth the skeleton data using a sliding time window. Specifically, we manually set the width of a time window to $T$ and then average the skeleton coordinates inside the time window. The time window keeps moving forward with stride of $T$ and the skeleton data in each time window will be averaged. In our system, we set $T$ to $200 \mathrm{~ms}$ which we empirically found to work well.

Anomaly filtering: We also found that occasional erroneous snapshots are reported, whose coordinates are jarringly different from previous and subsequent snapshots. We removed these unrealistic anomalous snapshots with a simple statistical method. For each time window of size $T$, we computed the average snapshot, then calculated the distance of each snapshot to the averaged snapshot, and assume the distances follow the normal distribution. The snapshots with a distance more than $3 \sigma$ to the average snapshot were removed, where $\sigma$ is the standard deviation of the normal distribution.

After applying these two operations to the data, all movements are smooth and continuous. Note that this data preprocessing need only be conducted on skeleton data for the trained (hemiparetic) body parts, which is of our interest. For example, if the left arm is being trained, we only smooth the left hand, wrist, elbow, and shoulder joints. With the above preprocessing techniques, we obtain a less fine-grained but more smooth and clean skeleton dataset.

\subsection{Contextual Filtering}

While we can analyze participants' motor performance during the whole period of game-play, sometimes it might be more useful to drill down to certain player contexts. We consider three different types of context in this work. Firstly, gesture context refers to different types of gestures, each of which corresponds to one kind of predefined movement. Secondly, different game regions can also be viewed as different region contexts, in which the users is required to conduct movement of various intensities. For example, it might be useful to know which game environments (e.g., self-paced versus fast-paced movements promoted) result in the best performance for each participant. Finally, the entire rehabilitation period consists of many continuous sessions of gameplay and we seek to understand the changes of performance during each such session context, as well as across different sessions (e.g., to detect fatigue). We segment across each of these contexts as described below.

Gesture segmentation: In addition to studying the skeleton data across all the gestures, we also segment the data by gestures and analyze the user's movement in each gesture. Our rehabilitation system contains 12 coded gestures (shoulder flexion, shoulder adduction, shoulder abduction, elbow flexion, elbow extension, etc.), which are mapped to varying game mechanics. Different gestures involve non-overlapping (and non-comparable) movement trajectories. Because of this, analytics on the segmented skeleton data need to account for what gesture is being attempted.

The game system records the timestamps of when various gestures (game mechanics) are triggered [11]. However, for the purpose of kinematic analysis, we are more interested in a gesture period, which is defined as a period of time that the user continuously conducts the same gesture. This is because a single snapshot will be static and only multiple continuous snapshots in a period can form dynamic movements, making kinematic analysis possible. The onset of each gesture is assumed to precede a game mechanic by no more than 2,000 ms because participants were consistently able to perform each gesture within this time frame. We eliminate movement epochs that did not trigger a game mechanic within this time window to eliminate movements that were not associated with a game mechanic. We then define the initial and final states of a gesture from the skeletal data based on the local minima and maxima of each movement. For example, the initial state of the shoulder abduction gesture would be identified as occurring when the angle in the xy plane between the arm and trunk was at a minimum (arm at side), whereas the final state of this gesture would occur when the angle in the xy plane between the arm and trunk was at a maximum (arm raised to the side to the greatest extent possible).

We then eliminate those isolated snapshots and figure out the beginning and ending time of each gesture period. We finally synchronize the timestamps between each gesture period and the skeleton data and so that the skeleton log entries can be matched to their corresponding gestures. By doing this, we are able to isolate the skeleton data for each type of therapeutic movement (e.g., shoulder flexion).

Game Region Segmentation: The game contains different "regions" of the river that promote different pace of play (e.g., self-paced versus fixed fast pace) and different distributions of gestures. Therefore, it can be useful to determine which motor training conditions promote the best motor performance. To contrast client performance between regions, the kinematics of different gestures in different regions should be studied independently. The game log generated by the software includes the timestamp of each region 
and we can extract the skeleton data of each region by synchronizing the game log region time with the skeleton log.

Session Segmentation: During in-home treatment, a user typically breaks the prescribed gameplay into several intervals throughout the day. We define a session as a continuous period of game-play without interruption longer than 10 minutes. To segment sessions, we mainly look at the game log. If there is no gesture detected in a continuous period of 10 minutes, we mark it as the end of the previous session and initiate a new game session. Skeleton data are then divided into sessions by synchronizing timestamps with game data. By segmenting the sessions, we can analyze the trajectory of motor performance within the same session, which might indicate gradual improvement or fatigue. Difference of motor performance across sessions, on the other hand, might be helpful for showing changes over the long term.

\subsection{Rehabilitation Efficacy Analysis}

We seek to study the participant's movement in the game throughout the rehabilitation period and to evaluate the rehabilitation efficacy. To this end, we propose a series of kinematic variables that help to measure participant's motor performance and assess the participant's movement impairments from different perspectives.

Hand Speed: People with hemiparesis often perform movements more slowly. Motor speed was identified by the stroke community as a priority clinical outcome because it indicates the extent to which tasks of daily living can be performed quickly and efficiently. Hereby, we adopt hand speed to capture motor performance. To calculate hand speed, we accumulate the distance that the hand moves in every two contiguous snapshots, and divide the distance by the accumulated time. Hand speed is sensitive to the angular range of excursion at both the shoulder and the elbow. Let $\vec{p}_{i}=\left(x_{i}, y_{i}, z_{i}\right)$ denote the coordinate of the hand in snapshot $i$ with timestamp $t_{i}$. Then given a series of snapshots at $t_{1}, t_{2}, \ldots, t_{n}$, we can get the hand speed by using

$$
v_{d}=\frac{\sum_{i=2}^{n}\left\|\vec{p}_{i}-\vec{p}_{i-1}\right\|}{\sum_{i=2}^{n}\left(t_{i}-t_{i-1}\right)} .
$$

Angle Speed: Angle speed is another way to measure speed of movement, specific to one joint, e.g., elbow or shoulder. It can be calculated in a similar way as hand speed, except that calculating the size of the angle requires three points from each snapshot: a joint point and two other end points. For example, to calculate the angle of the elbow, we can use the elbow as the joint point, and then wrist and shoulder as end points. Let $\vec{r}_{i}=\left(x_{1 i}, y_{1 i}, z_{1 i}\right)$ denote coordinate of the joint point, and $\vec{s}_{i}=\left(x_{2 i}, y_{2 i}, z_{2 i}\right)$ and $\overrightarrow{t_{i}}=\left(x_{3 i}, y_{3 i}, z_{3 i}\right)$ denote coordinates of two other end points at time $t_{i}$. Denoting the joint angle at $t_{i}$ as $\alpha_{i}$, then

$$
\alpha_{i}=\arccos \frac{\left(\vec{s}_{i}-\vec{r}_{i}\right) \cdot\left(\vec{t}_{i}-\vec{r}_{i}\right)}{\left\|\vec{s}_{i}-\vec{r}_{i}\right\|\left\|\vec{t}_{i}-\vec{r}_{i}\right\|} .
$$

And therefore, the angle speed can be calculated as

$$
v_{a}=\frac{\sum_{i=2}^{n}\left|\alpha_{i}-\alpha_{i-1}\right|}{\sum_{i=2}^{n}\left(t_{i}-t_{i-1}\right)}
$$

Range of Motion: A participant's range of motion is defined as the difference between the maximum and minimum joint angles recorded during the rehabilitation gaming. Range of motion measures the active range of motion of the body joint. To figure out the range of motion of a joint, we calculate the joint angle of each snapshot during a game session using Equation 2 and sort those values in ascending order, denoted as $\alpha_{(1)}, \ldots, \alpha_{(n)}$. To correct for outliers at the extremes of the joint angles, we increase the robustness of the range of motion value $R$ by subtracting the $k$-th smallest from the $k$-th largest joint angle, i.e.

$$
R=\alpha_{(n-k+1)}-\alpha_{(k)}
$$

where $k$ is set as $5 \% * n$ in this paper, which we empirically found to work well.

While the above kinematic variables could be applicable to all skeletal joints, we have restricted our analyses to the hemiparetic (weaker) extremity of our MS participants. By looking at these kinematic variables throughout the rehabilitation period, we are provided with a continuous, objective measure of rehabilitation progress.

\section{EXPERIMENT AND ANALYSIS}

In this section, we apply our data analytics framework on data collected from in-home game play of participants. We recruited participants from the local demographic of individuals with mild to moderate hemiparesis due to multiple sclerosis (MS). Participants were loaned a gaming system and agreed to play the game for 1.5 hours daily over a 3 week period. Participants received an initial consultation session with a therapist to learn how to operate the game. The game course was customized to the participant's motor objectives by the therapist during the first study visit. Three additional in-home visits were conducted to promote adherence to the protocol and carryover of motor gains to daily activities.

Table 1: Basic Information of Participants

\begin{tabular}{|l|l|l|l|l|}
\hline Participant ID & Affected Side & Days & Total Mins & Avg Mins/Day \\
\hline P1 & Left & 21 & 678.6 & 32.3 \\
\hline P2 & Right & 14 & 803.2 & 57.4 \\
\hline P3 & Left & 19 & 656.0 & 34.5 \\
\hline P4 & Right & 21 & 978.5 & 46.6 \\
\hline P5 & Right & 20 & 2185.5 & 109.3 \\
\hline P6 & Right & 21 & 2084.3 & 99.3 \\
\hline P7 & Right & 20 & 1351.4 & 67.6 \\
\hline P8 & Right & 13 & 881.6 & 67.8 \\
\hline
\end{tabular}

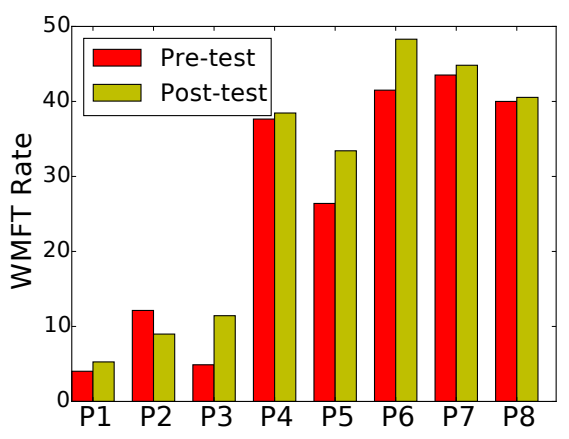

Figure 3: Pre/post comparison: the Wolf Motor Function Test result from the lab.

Participants' rehabilitation data is electronically captured by the software during live gameplay and stored on each participant's machine. The data acquired consists of skeleton 


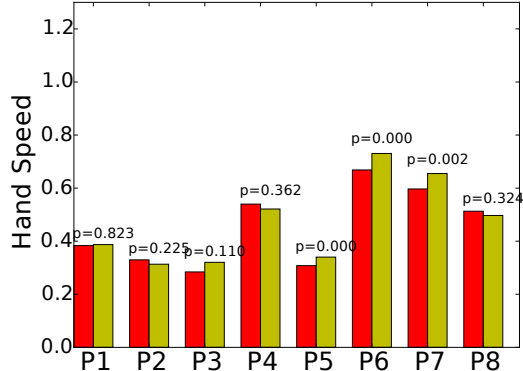

(a) Hand Speed

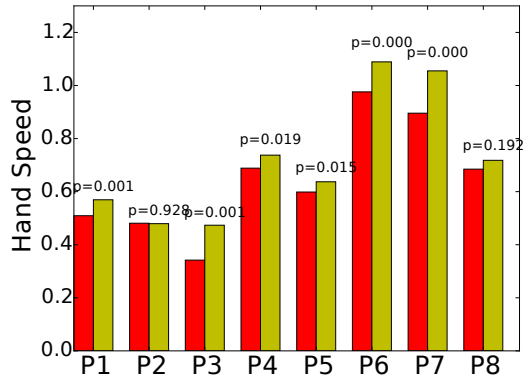

(d) Hand Speed

(raise-arm-side gesture)

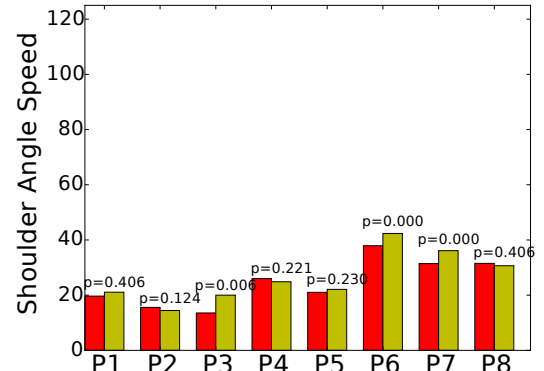

(b) Shoulder Angle Speed

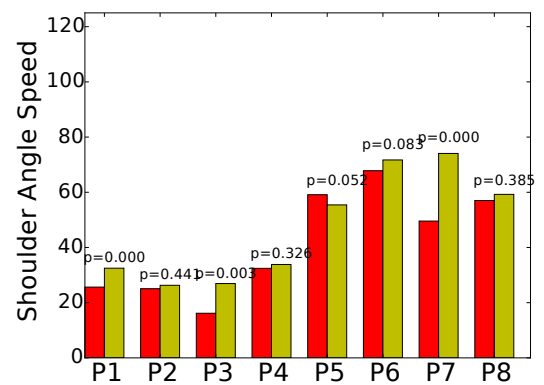

(e) Shoulder Angle Speed (raise-arm-side gesture)

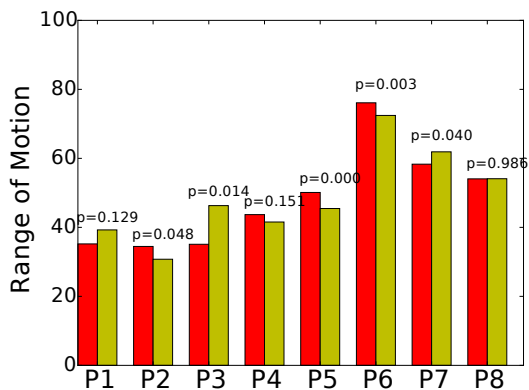

(c) Shoulder Range of Motion

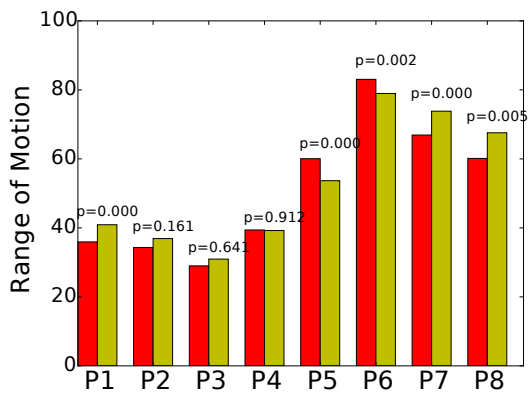

(f) Shoulder Range of Motion (raise-arm-side gesture)

Figure 4: Two-half comparison: Kinematic analysis.

data from the Kinect camera tracking system and the game $\log$ from the software.

Before and after the participants use the rehabilitation system, the Wolf Motor Function Test (WMFT) [5] was administered to them in the lab. The test measures the time to perform 15 standardized motor tasks, e.g., lifting pencil and placing the hand on top of a box. The time to complete each task is converted to a WMFT rate score by dividing 60 seconds by the completion time for each task. The WMFT rate metric represents the average WMFT rate across all 15 tasks. We present here an initial comparison between change in the kinematic variables proposed and this laboratory-based measure.

The first eight participants have finished using the rehabilitation system and we use data collected from them for a detailed analysis. Data for more participants was still being collected at the time of this submission.

The basic information of these eight participants is shown in Table 1. As the table shows, six have right upper extremity hemiparesis and two have left upper extremity hemiparesis. We conduct temporal smoothing and filter out anomalies following the data preprocessing techniques mentioned in Section 3.1. We now employ the proposed kinematic variables in Section 3.3 to study the efficacy of our rehabilitation system.

\subsection{Two-half Comparison of Kinematic Vari- ables}

In order to analyze the efficacy of rehabilitation, we adopted a two-half comparison approach. We divided the whole re- habilitation period into two halves, i.e. the first half and the second half. We calculate the kinematic variables in these two divisions respectively, and analyze their differences. Specifically, for a kinematic variable on a body part, we figure out the average value every minute respectively in the first and second half of rehabilitation period. With these data points in each half, we further average the values in the same half and conduct $t$-test for the two halves in order to examine whether their averaged values are significantly different.

To assess the motor performance of users, we adopt the kinematic variables proposed in Section 3.3. For our initial metric to demonstrate the feasibility of game rehabilitation system, we specifically chose hand speed, angle speed of the shoulder and range of motion of the shoulder on the more affected side.

We use the two-half comparison approach on these kinematic variables for the eight users. Figures $4 \mathrm{a}, 4 \mathrm{~b}$ and $4 \mathrm{c}$ show the two-half comparison of the three kinematic variables, where the $p$ value of each $t$-test is also reported. We also drill down to single gesture to study the motor performance under one gesture. Figures $4 \mathrm{~d}-4 \mathrm{f}$. show twohalf comparison results for raise-arm-side gesture. Moreover, we adopt the WMFT rate metrics obtained pre- and post-treatment to cross-reference with the kinematic findings (Figure 3). We discuss below observations obtained from these figures.

Motor Performance Strength. Note that the motor performance represented by our kinematic variables is highly consistent with the WMFT rate metric obtained in the lab- 


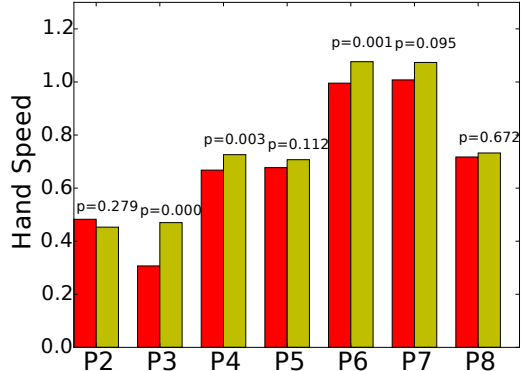

(a) Hand Speed (raise-arm-side in Rowing Region)

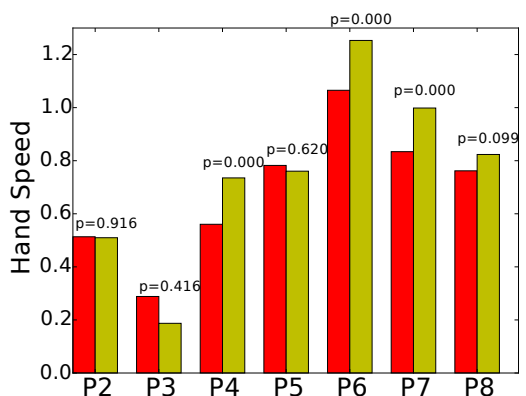

(d) Hand Speed (raise-arm-side in Rapids Region)

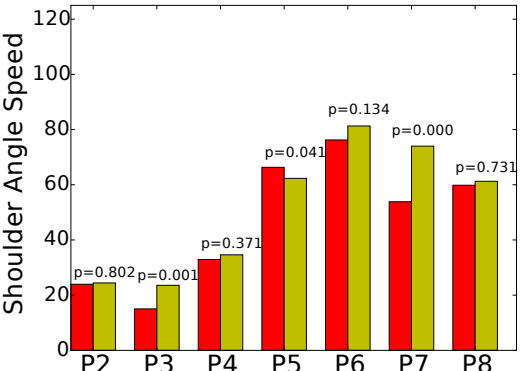

(b) Shoulder Angle Speed (raise-arm-side in Rowing Region)

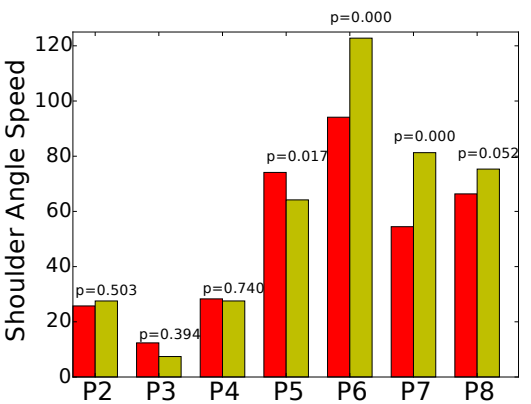

(e) Shoulder Angle Speed (raise-arm-side in Rapids Region)

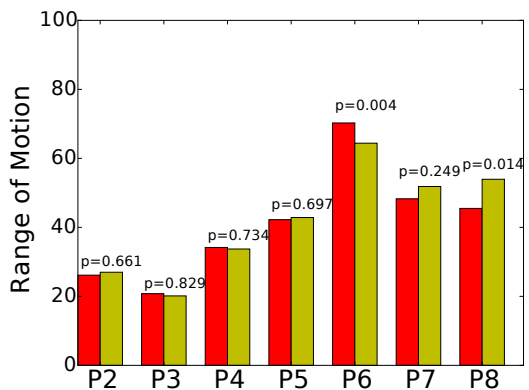

(c) Shoulder Range of Motion (raise-arm-side in Rowing Region)

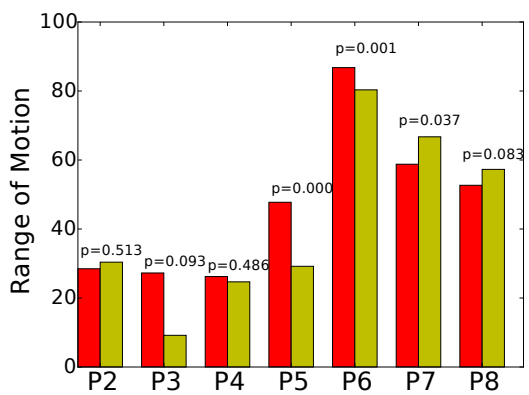

(f) Shoulder Range of Motion (raise-arm-side in Rapids Region)

Figure 5: Two-half comparison on Rowing and Rapids region respectively. (a)-(c) are two-half comparison based on data merely from Rowing region. (d)-(f) are based on Rapids region.

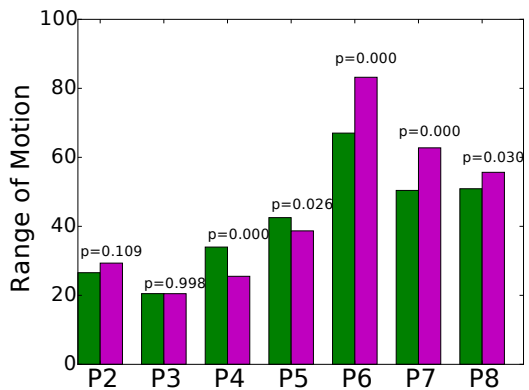

(c) Shoulder Range of Motion (raise-arm-side)

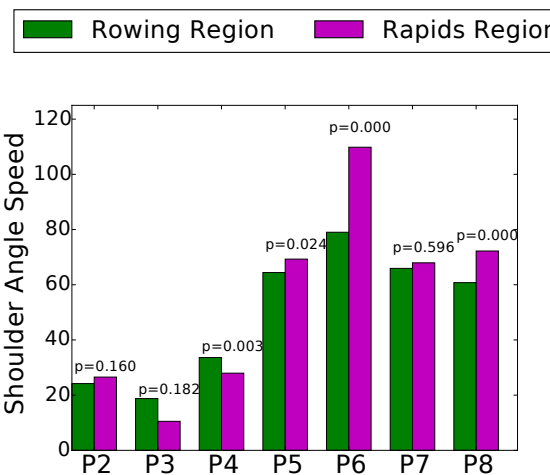

(b) Shoulder Angle Speed (raise-arm-side) (a) Hand Speed (raise-arm-side)

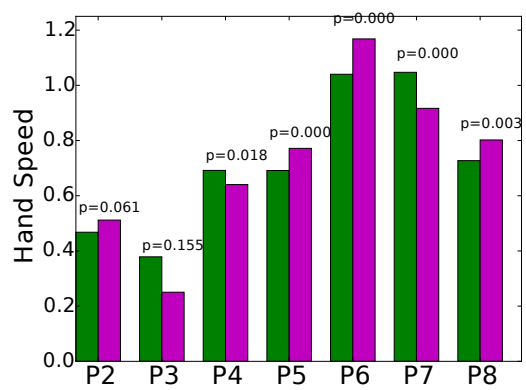

Figure 6: Kinematic measurements comparison between Rowing region and Rapids region. oratory for both the speed and range of motion metrics (Figure 3 cross-referenced with Figure 4). The participants with higher WMFT rates in pre-test and post-test usually perform better in the game with higher values in the kinematic values. Specifically, we observe that P1, P2 and P3 get much lower WMFT rates than P6, P7 and P8 according to Figure 3. This is highly consistent with the plots in Figure 4, the majority of which show $\mathrm{P} 6, \mathrm{P} 7$ and $\mathrm{P} 8$ perform better than P1, P2 and P3. This consistency is most pronounced for hand speed in Figures 4a and 4d where the order of kinematic values of participants is almost the same as in Figure 3.

Improvement Trend and Scale. According to Figure 3, P3, P5 and P6 have substantial improvement on WMFT rate after the rehabilitation. The kinematic analysis in the game shows similar results. Figures $4 \mathrm{a}, 4 \mathrm{~b}$ and $4 \mathrm{~d}$ show that P3, P5 and P6 have significant improvements over the two periods with small $p$ value. The improvement of $\mathrm{P} 6$ on hand 


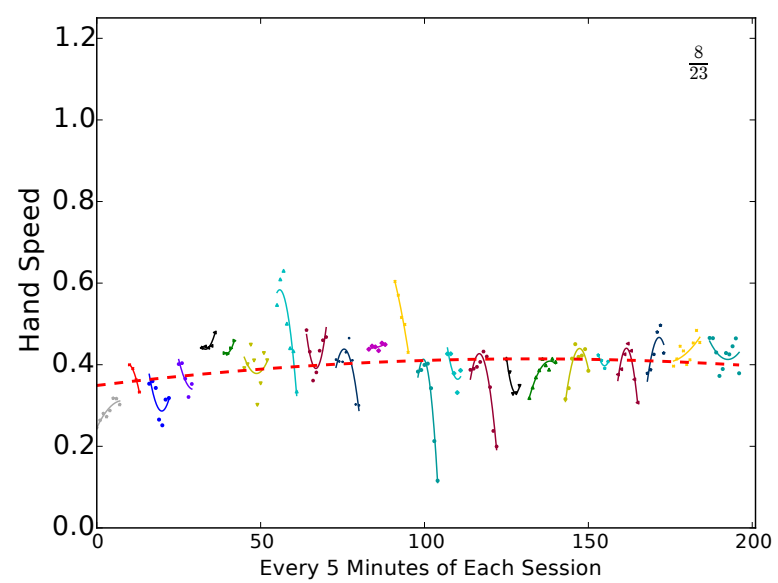

(a) P1

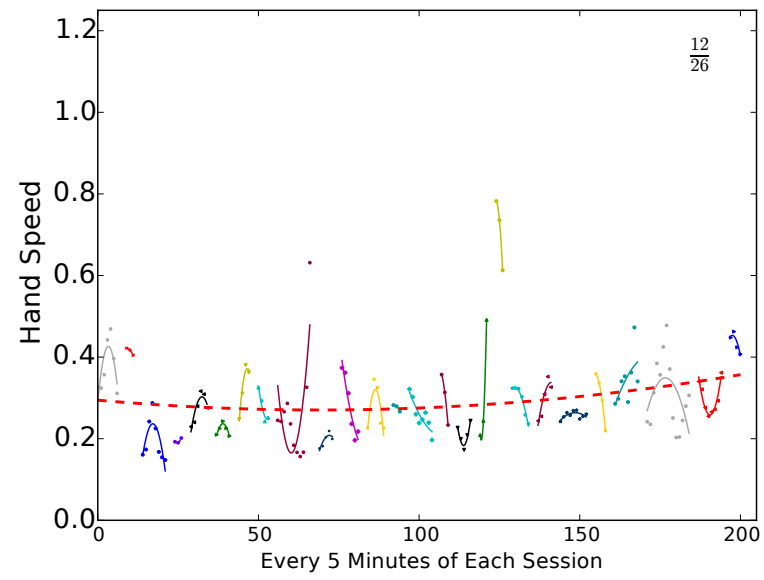

(c) P3

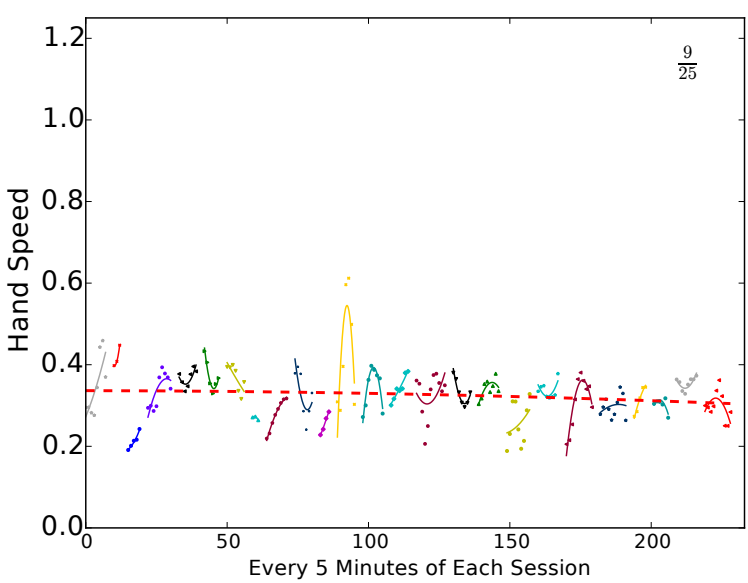

(b) P2

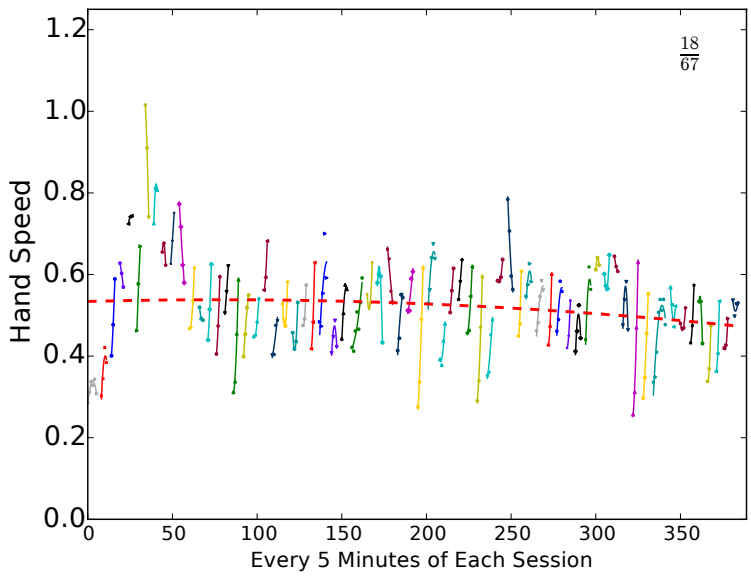

(d) $\mathrm{P} 4$

Figure 7: Hand speed trend of each session. Colored real lines associated with colored points are results of quadratic regression. Red dash lines are quadratic regression for all the data points. Each number in the upper-right corner is the number of inverted "U" over total number of sessions.(P1-P4)

speed and shoulder angle speed is particularly high with $p$ value smaller than 0.002 . In addition, $\mathrm{P} 1, \mathrm{P} 4, \mathrm{P} 7$ and $\mathrm{P} 8$ achieve marginal gain over the rehabilitation period. Consistent with this, P3, P5, and P6 make significant gains on the WMFT rate metric, whereas no significant improvement is observed in Figure 3 for participants P1, P4, P7, and P8. P2 showed decreased WMFT rate in Figure 3 and degraded performance in Figure 4a-4c.

It is surprising that though WMFT rate measures the performance of a variety of different gestures, our kinematic measurement of just one gesture shows a consistent pattern of performance. Note that WMFT is quite expensive to conduct since it requires the participants to show up in person and perform a series of tasks, while our kinematic measurement can be obtained remotely without a trained tester and can measure the change of performance in real time across the whole rehabilitation period (instead of just pre/post treatment). Therefore, kinematic measurement based on gameplay data log can serve as a complimentary way to conveniently assess participants' initial motor deficit and mon- itor rehabilitation progress.

Furthermore, we can observe that the majority of the participants have obvious improvement in hand speed, shoulder angle speed and range of motion of the shoulder during the rehabilitation. However, different individuals might benefit from our rehabilitation system in different ways. Some participants show similar improvement over all the kinematic measurements under various gestures, such as P1 and P3. Some participants show improvements on different kinematics measurements, including $\mathrm{P} 4, \mathrm{P} 5, \mathrm{P} 6, \mathrm{P} 7$ and P8. Others (e.g., P2) did not have obvious improvements for most of the measurements. In particular, we notice that some participants achieved greater speed of movement (e.g., P6 and P7), but not improved range of motion. This is further evidence of the need to capture recovery throughout an intervention using kinematic variables that can more specifically isolate various patterns of motor recovery. Existing clinical motor metrics are unable to isolate the independent contributions of motor speed and active range of motion on overall performance. 


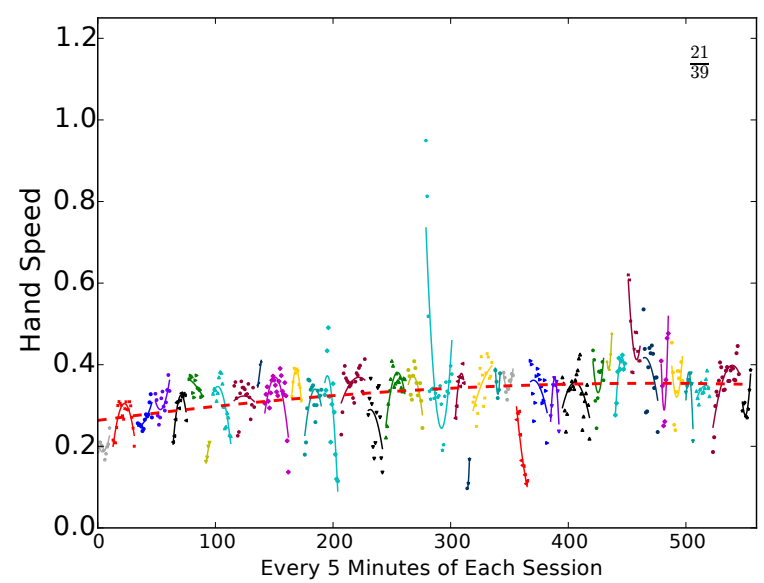

(a) P5

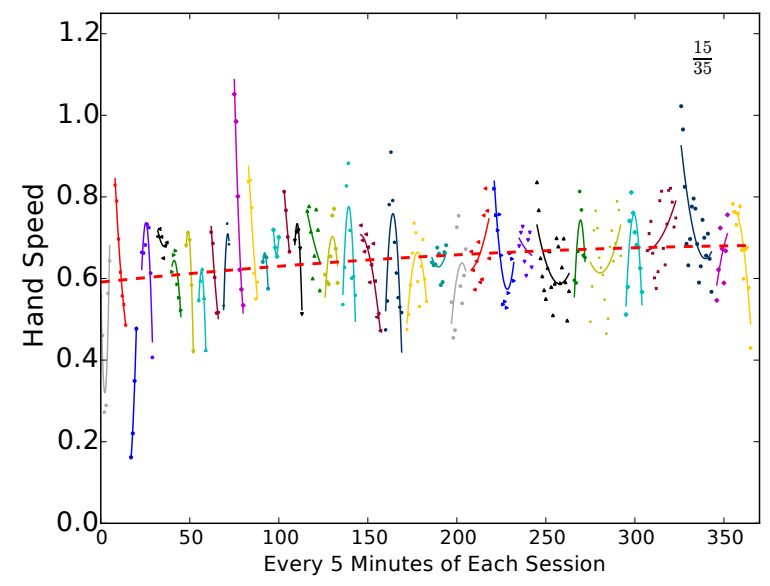

(c) P7

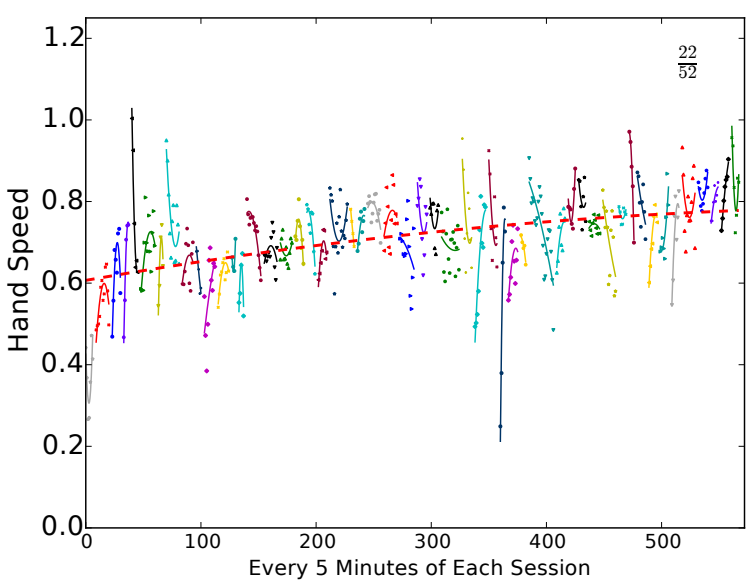

(b) P6

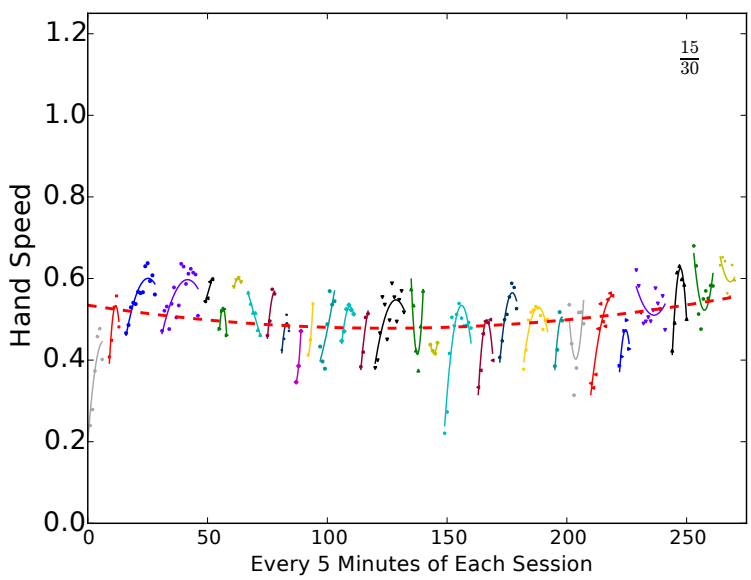

(d) P8

Figure 8: Hand speed trend of each session. Colored real lines associated with colored points are results of quadratic regression. Red dash lines are quadratic regression for all the data points. Each number in the upper-right corner is the number of inverted "U" over total number of sessions.(P5-P8)

\subsection{Region Segmentation}

We filter the game log by regions using the segmentation techniques introduced in Section 3.2. We specifically study two common regions: Rowing region and Rapids region. The Rowing region of the game allows the user to move at his/her own pace, while the Rapids region pushes the user to react at a fast pace that is set by the game. Furthermore, we merely focus on the raise-arm-side gesture which is a key movement for navigation, and is thus utilized frequently within the game. Results are shown in Figure 5. From the plots in Figure 5, we observe analogous phenomenon as above ${ }^{2}$.

Contrary to expectation, we found that some participants did not move significantly faster in the Rapids region than the Rowing region overall. To better understand this, we separate the data into Rowing region and Rapids region and figure out the average kinematic measurement values under raise-arm-side gesture. Similar to two-half comparison, T-

${ }^{2} \mathrm{P} 1$ is not shown since their game log does not include regions in the game-play. test is conducted. The results are shown in Figure 6. We can observe that the majority of participants performed larger movements during fast-paced play than during self-paced play (P2, P5, P6 and P8), whereas others performed better under self-paced game conditions (P3, P4 and P7).

\subsection{Fine-grained Trend Analysis}

We now conduct a fine-grained trend analysis. We intend to study the change of kinematic measurements in each session and across sessions in the whole rehabilitation period. We use the techniques introduced in Section 3.2 to segment each session. We then study the hand movement speed of the participants every five minutes in each session so that we can observe the change of participant's performance. Figures 7 and 8 show the trend of hand speed in all participants, where each dot shows the averaged speed during the five minutes and each curve segment represents the trend of speed in that session.

We notice that for many participants, kinematic measurement values are more frequently decreasing at the end of 
a session of play. In particular, it is common to observe a decrease after an initial increase inside a session (inverted 'U' shape). However, participant 4 consistently performed faster movements toward the end of each play session. Similar patterns occur in analysis of angle speed and range of motion (figures are omitted due to space limitations). We hypothesize that the pattern of decreasing trends after initial increase, i.e. the inverted "U" shape, may be indicative of fatigue [15]. Driven by this observation, we adopt a simple way to evidence the existence of diminishing performance after a period of time.

For the data points in each session, we use quadratic regression to capture the trend of kinematic measurement values. The regression curves are shown in Figure 7 and Figure 8 along the scatter plots. We also conduct a global quadratic regression for all the data points in order to provide the holistic trend, which are shown as red dashed lines across the plots. Since we are particularly interested in the inverted 'U' shape trend, we identify sessions with this shape by looking at each session's quadratic regression function. This is easily performed by checking that the sign of coefficient of squared term is negative and the position of axis of symmetry is inside the interval. In the upper-right corner of each plot in Figure 7 and 8, we show the number of inverted ' $U$ ' sessions over the total number of sessions (i.e. the number of colored curves).

We highlight the following interesting observations:

- Overall trend is consistent with two-half comparison. We can observe that the overall trend (the global quadratic, red-dashed line) is quite consistent with Figure 4. P2 and P4, for whom we observe a decrease in the two-half analysis in Figure 4, go downward in the global regression curves. P5, P6 and P7, which present significant increase in two-half analysis, show a steady upward trend in Figure 8a- 8c.

- Fatigue is potentially related to the amount time of gameplay. In support of our hypothesis that the inverted ' $U$ ' trend is indicative of fatigue, this trend appears to be related to the amount of time the users spend in a session of gameplay. It is easy to see that $\mathrm{P} 5-\mathrm{P} 8$, who have longer duration sessions than P1-P4 (see Table 1), have many more inverted 'U' sessions in the trend. Objective fatigue detection, as may be possible here, has many promising applications for understanding the relationship between fatigue and rehabilitation progress and for gaining a better understanding of the origins of fatigue.

\section{CONCLUSION}

In this paper, we propose a data analytics framework for our game-based rehabilitation system. Data processing techniques and several kinematic metrics for efficacy analysis are discussed. We employ our data analytics framework to kinematic data captured during in-home game play to reveal the efficacy of the therapy across different aspects of motor performance. We also propose a method that may prove to capture fatigue through fine-grained trend analysis.

In future work, we plan to apply our data analytics framework to future participants in two different clinical trials. This data will provide more time-sensitive information on dose-response than can be captured with standard pre-post clinical measures (like the WMFT). We also intend to study the efficacy of our system on individuals over a longer period (one or two years). We plan to incorporate additional kinematic metrics to evaluate the efficacy of the rehabilitation more comprehensively. Furthermore, we envision operating this data analytics framework in real time while the participant is playing the game to provide real-time feedback and to personalize the therapy experience, e.g., to detect fatigue in real time and adjust game play pace accordingly.

Acknowledgments: This work was supported in part by the National Multiple Sclerosis Society, Participant-Centered Outcomes Research Institute (PCORI), UL1TR001070 from the National Center For Advancing Translational Sciences, NSF-EAR-1520870 and NSF-DMS-1418265 from National Science Foundation.

\section{REFERENCES}

[1] Y.-J. Chang, S.-F. Chen, and J.-D. Huang. A kinect-based system for physical rehabilitation: A pilot study for young adults with motor disabilities. Research in Developmental Disabilities, 32(6):2566-2570, 2011.

[2] F. de NAP Shelton and M. J. Reding. Effect of lesion location on upper limb motor recovery after stroke. Stroke, 32(1):107-112, 2001.

[3] C. for Disease Control, P. (CDC, et al. Prevalence of disabilities and associated health conditions among adults-united states, 1999. MMWR. Morbidity and mortality weekly report, 50(7):120, 2001.

[4] Heart and S. F. of Ontario. A report from the consensus panel on stroke rehabilitation system. 2001.

[5] T. M. Hodics, K. Nakatsuka, B. Upreti, A. Alex, P. S. Smith, and J. C. Pezzullo. Wolf motor function test for characterizing moderate to severe hemiparesis in stroke patients. Archives of physical medicine and rehabilitation, 93(11):1963-1967, 2012.

[6] M. Kelly-Hayes, A. Beiser, C. S. Kase, A. Scaramucci, R. B. D'Agostino, and P. A. Wolf. The influence of gender and age on disability following ischemic stroke: the framingham study. Journal of Stroke and Cerebrovascular Diseases, 12(3):119-126, 2003.

[7] B. Lange, S. Koenig, C. Chang, E. McConnell, E. Suma, M. Bolas, and A. Rizzo. Designing informed game-based rehabilitation tasks leveraging advances in virtual reality. Disabil Rehabil., 34(22):1863-70, 2012.

[8] B. Lange, S. Koenig, E. McConnell, C. Chang, R. Juang, E. Suma, M. Bolas, and A. Rizzo. Interactive game-based rehabilitation using the microsoft kinect. In Virtual Reality Short Papers and Posters (VRW), 2012 IEEE, pages 171-172. IEEE, 2012.

[9] D. Lloyd-Jones, R. Adams, M. Carnethon, G. De Simone, T. B. Ferguson, K. Flegal, E. Ford, K. Furie, A. Go, K. Greenlund, et al. Heart disease and stroke statistics - 2009 update a report from the american heart association statistics committee and stroke statistics subcommittee. Circulation, 119(3):e21-e181, 2009.

[10] D. Maung, R. Crawfis, L. V. Gauthier, L. Worthen-Chaudhari, L. P. Lowes, A. Borstad, and R. J. McPherson. Games for therapy: Defining a grammar and implementation for the recognition of therapeutic gestures. In FDG, pages 314-321, 2013. 
[11] D. Maung, R. Crawfis, L. V. Gauthier, L. Worthen-Chaudhari, L. P. Lowes, A. Borstad, R. J. McPherson, J. Grealy, and J. Adams. Development of recovery rapids-a game for cost effective stroke therapy. In Proceedings of the International Conference on the Foundations of Digital Games, 2014.

[12] V. L. Roger, A. S. Go, D. M. Lloyd-Jones, E. J. Benjamin, J. D. Berry, W. B. Borden, D. M. Bravata, S. Dai, E. S. Ford, C. S. Fox, et al. Executive summary: heart disease and stroke statistics-2012 update: a report from the american heart association. Circulation, 125(1):188, 2012.

[13] C.-J. Su et al. Personal rehabilitation exercise assistant with kinect and dynamic time warping. IJIET, 3(4):448-454, 2013.

[14] S. L. Wolf, C. J. Winstein, J. P. Miller, E. Taub, G. Uswatte, D. Morris, C. Giuliani, K. E. Light, D. Nichols-Larsen, et al. Effect of constraint-induced movement therapy on upper extremity function 3 to 9 months after stroke: the excite randomized clinical trial. Jama, 296(17):2095-2104, 2006.

[15] N. Yozbatiran, F. Baskurt, Z. Baskurt, S. Ozakbas, and E. Idiman. Motor assessment of upper extremity function and its relation with fatigue, cognitive function and quality of life in multiple sclerosis patients. Journal of the neurological sciences, 246(1):117-122, 2006. 